\title{
Optimum Dispatch of Hybrid Solar Thermal (HSTP) Electric Power Plant Using Non-Smooth Cost Function and Emission Function for IEEE-30 Bus System
}

\author{
http://dx.doi.org/10.3991/ijes.v4i2.5699 \\ Dash Saroj Kumar, Ray Priyambada, Panda Chinmaya Kumar \\ GITA, Bhubaneswar, Odessa, India
}

\begin{abstract}
The basic objective of economic load dispatch (ELD) is to optimize the total fuel cost of hybrid solar thermal electric power plant (HSTP). In ELD problems the cost function for each generator has been approximated by a single quadratic cost equation. As cost of coal increases, it becomes even more important have a good model for the production cost of each generator for the solar thermal hybrid system. A more accurate formulation is obtained for the ELD problem by expressing the generation cost function as a piece wise quadratic cost function. However, the solution methods for ELD problem with piece wise quadratic cost function requires much complicated algorithms such as the hierarchical structure approach along with evolutionary computations (ECs). A test system comprising of 10 units with 29 different fuel [7] cost equations is considered in this paper. The applied genetic algorithm method will provide optimal solution for the given load demand.
\end{abstract}

Index Terms-Economic Load Dispatch (ELD), Combined Cycle Plant(CCP), Genetic Algorithm(GA),Hybrid Solar Thermal Power Plant(HSTP), Evolutionary Computations(ECs),Multi-fuel Effects(MFE)

\section{INTRODUCTION}

The basic theme of economic dispatch is to determine the optimal combination of power outputs of the generating units in electric power system so as to optimize the total fuel cost for a certain load demand satisfying operational constraints. The economic load dispatch (ELD) problem is analyzed basically through the input output characteristic or through the heat rate input output characteristic by taking real power output of ith generating unit (PGi) in the $\mathrm{X}$ axis and Fuel input in rupees per hour in the $\mathrm{Y}$ axis. Input-Output characteristic is approximated as a single quadratic variation curve which gives sub-optimal solutions. Usually the nature of Input-Output characteristics of modern generating units is non-linear because of multi-fuel effects (M F E) using combined cycle power plants (CCPP) and valve loading effects, which may lead to multiple local minimum points of cost functions. Hence it is more realistic to represent the Input-Output characteristic as a piece wise quadratic cost function to avoid huge revenue loss over time problems. This project develops algorithm approach for solving the economic dispatch problem for a test system of 10 plants having 29 fuel cost options. A salient feature of proposed approach is that solution time grows approximately linear with problem size. More over the inclusion of solar power plant in tandem with the thermal power plant reduces the emission level so as to maximize the power generation for solar plant leading to minimum utility of the solar generation. The quadratic cost function so chosen is minimized following the reduction in emission level of Sulphur dioxide (SO2), carbon monoxide (CO), nitrous oxide $\mathrm{N} 2 \mathrm{O}$ and other greenhouse gasses. The quadratic programming approach so chosen for the hybrid solar thermal power system optimizes the cost of generation of solar thermal power plant and simulation time as well.

\section{ECONOMIC LOAD DISPATCH}

The ELD [3] problem is to determine the optimal combination of power outputs of all the generating units to minimize the total fuel cost while satisfying the load demand from the operational constraints. Minimum fuel costs are achieved by the economic load scheduling of different generating units. Here we mean to ascertain the generation of distinct generators so as to obtain the total fuel cost as minimum so that the load demand is met out by net generation.

\section{A. Economic Load Dispatch Problem}

However, economic load scheduling was not of relevance when there were small power generating plants for each locality, such as urban power system, but now with the growth in the power demand and at the same time guarantee regarding the continuity of power supply to the consumer under normal conditions have forced the power system engineers to develop grid system. For such system the economic dispatch problem has become increasingly important. The objective in the economic dispatch of power system is to minimize the cost of meeting the energy requirements of the system over some appropriate period of time and in a manner consistent with reliable service. The appropriate period may be as short as few minutes or as long as a year or more depending on the nature of the energy sources available to the system.

\section{B. Problem Statement for ELD with Non-Smooth Cost}

\section{Function}

Let $\mathrm{N}$ be the number of units.

$$
P G_{i} \text { be the power supplied by the } i_{\text {th }} \text { unit. }
$$$$
\mathrm{PD} \text { be the load demand in MW }
$$ 
The generation cost objective function for the thermal power plant in proposed method can be represented by cost function [2]:

$$
f_{1}(P G)=\sum_{i=1}^{N G} a_{i} P G_{i}^{2}+\lambda\left|e i \sin \left(P G_{i}+c_{i} \min -P G_{i}\right)\right| R s / h
$$

Where, ${ }^{a_{i}}, b_{i}$ and $c_{i}$ are generation cost coefficients for the $i_{\text {th }}$ generating[4] unit subjected to condition

$\sum P G i=P_{D}+P_{L}$ Where $i=1,2,3 \ldots \ldots . . n$

$P_{L}=\sum_{m=1}^{n} \sum_{n=1}^{n} P G_{m} P G_{n} B_{m n}$

$\sum B_{m n}=\frac{\cos \left(\sigma_{m}-\sigma_{n}\right) M p_{m} \times M p_{n} \times P_{L}}{\left|V_{m}\right| \times\left|V_{n}\right| \cos \phi_{m} \times \cos \phi_{n}}$

Neglecting valve point loading [3] and incorporating transmission loss using conventional method the cost of thermal generation is expressed as

$$
\left.\begin{array}{c}
F(P G)=\sum_{2} a_{i} P G_{i}^{2}+b_{i} P G_{i}+c_{i}+\lambda\left(P_{D}+P_{L}-\sum P G_{i}\right) \\
f_{i}\left(P_{i}\right)=\sum_{i=1}^{N G} \alpha_{i} P G_{i}^{2}+\beta_{i} P G_{i}+\gamma_{i}+\eta_{i} \exp \left(-k_{i} P G_{s}\right) R s / h
\end{array}\right\}
$$

$$
\text { Subject to condition } \mathrm{PGi}(\min ) \leq P G i \leq \mathrm{PGi}(\max )
$$

\section{Cost Criteria for Economic Load Dispatch Problem}

Using Lagrangian multiplier method fuel cost and emission cost functions incorporating transmission loss were expressed in equation (1) and equation (2) respectively.

By differentiating the above equations we get:

$$
\begin{aligned}
& \frac{d F(P G)}{d P G_{i}}=0 \\
& \Rightarrow 2 a_{i} P G_{i}+b_{i}+\lambda *\left(\frac{\partial P_{L}}{\partial P G_{i}}-1\right)=0 \& \\
& \frac{\partial F(P G)}{\partial \lambda}=P_{L}-\sum P G_{i}+P_{D} \\
& \sum P G_{i}=\left(P_{D}+P_{L}\right)
\end{aligned}
$$

\section{Dividing equation (7) by $2 a_{i}$}

$$
P G_{i}+\frac{b i}{2 a_{i}}+\lambda\left[\frac{\frac{\partial P_{L}}{\partial P G_{i}}-1}{2 a_{i}}\right]=0
$$

$$
\begin{gathered}
\Rightarrow P G_{i}+\frac{b_{i}}{2 a_{i}}=\frac{\lambda}{2 a_{i}}\left(1-\frac{\partial P_{L}}{\partial P G_{i}}\right)(10) \\
\Rightarrow \sum P G_{i}+\frac{\sum b_{i}}{2 a_{i}}=\lambda \sum \frac{1}{2 a_{i}}\left(1-\frac{\partial P_{L}}{\partial P G_{i}}\right) \\
\Rightarrow P G_{i}=\frac{\lambda\left(1-\frac{\partial P_{L}}{\partial P G_{i}}\right)-b_{i}}{2 a_{i}}
\end{gathered}
$$

\section{SOLAR THERMAL HYBRID PlANT}

$$
f_{2}\left(P_{i}\right)=\sum_{i=1}^{N G} \alpha_{i} P G_{i}^{2}+\beta_{i} P G_{i}+\gamma_{i}+\eta_{i} \exp \left(-k_{i} P G_{s}\right) R s / h
$$

Where, $\alpha_{i}, \beta_{i} \gamma_{i}$ and $\eta_{i}$ are generation cost coefficients for the $i_{\text {th }}$ generating unit subjected to condition

$f_{1}(P G)=\sum_{i=1}^{N G} a_{i} P G_{i}^{2}+b_{i} P G_{i}+c_{i}+\lambda \times f_{2}\left(P_{i}\right)\left|e i \sin \left(P G_{i \min }-P G_{i}\right)\right| R_{s} / h(13)$

\section{Genetic Algorithm}

Genetic algorithm (GA), was first propounded by John Holland in early seventies, is a flagship among various techniques of function optimization. Genetic algorithm criss-crosses all the above limitations of conventional algorithms by using the basic building blocks that are distinct from those of conventional algorithms. The following differential aspects are as follows:

1. GA works with a coding of the parameters set and not the parameters themselves.

2. GA searches from a population of points and not from a single point like conventional algorithm.

3. GA uses objective function information, not derivative or other auxiliary data.

4. GA uses probabilistic transition rules by stochastic operands, not by deterministic rules.

The initial step of GA [8] is the random selection of initial search points from the total search space. Each and every point in the search space corresponds to one set of values for the parameters of the problem. Each parameter is coded with a string of bits. The individual bit is called "gene". The content of each gene is called "allele". The total string of such genes of all parameters written in a sequence is called a "chromosome". So there exists a chromosome for each point in the search space. The sea of search points selected and used for processing is called a population. That means population is a set of chromosomes. The no of chromosomes in population is called "population size" and the total number of genes in a string is called "string length". The population is processed and evaluated through various operators of GA to generate a new population and this process is carried out till global 
PAPER

Optimum Dispatch of Hybrid Solar Thermal (HSTP) Electric Power Plant Using Non-Smooth Cost...

optimum point is reached. The two parts of the process are called "generation and evaluation".

For the evaluation of GA we define a fitness function and evaluate the fitness for each chromosome of a population. This fitness is an indication of the suitability of the values of the parameters, as represented by that chromosome and acts as a solution of the optimization problem [9] under consideration. This fitness is used as bias for selecting the parents and generating a new population from the existing one.

\section{Result AND PERformance CharaCteristic}

The various program specific arguments for the optimization of cost and emission function of hybrid

Solar thermal power plant were tabulated in the tables1,2 and 3.

The increment in solar generation is accompanied by decrement in emission level in the thermal power plant thereby optimizing the cost of generation of the thermal power plant as shown in Fig-1.

Average cost of generation incorporating cost of fuel for solar thermal power plant for various units of thermal power plant and various generating modules of solar power plant with respect to number of units of the hybrid plant decreases with number of units initially and rises further with further increment of number of units as shown in Fig-2.

TABLE I.

\begin{tabular}{|c|c|c|c|c|c|c|}
\hline $\begin{array}{c}\text { Power } \\
\text { genera- } \\
\text { tion } \\
\text { (PGi) }\end{array}$ & ai & bi & ci & $\begin{array}{c}\text { Power } \\
\text { demand } \\
\text { (PD) }\end{array}$ & ei & $\begin{array}{c}\text { Power } \\
\text { loss } \\
\text { (PL) }\end{array}$ \\
\hline 404.40 & 0.05 & 2.33 & 39.79 & 1870 & 134.21 & 12.51 \\
\hline 285.93 & 0.01 & 0.09 & 13.97 & 1900 & 153.95 & 39.74 \\
\hline 255.66 & 0.01 & 0.08 & 13.92 & 1930 & 155.98 & 73.89 \\
\hline 243.33 & 0.01 & 0.31 & 39.79 & 1960 & 157.23 & 49.68 \\
\hline 297.72 & 0.01 & 0.13 & 18.95 & 1990 & 161.63 & 61.18 \\
\hline 162.79 & 0.01 & 0.01 & 14.23 & 2020 & 240.01 & 125.95 \\
\hline
\end{tabular}

TABLE II.

\begin{tabular}{|c|c|c|c|c|}
\hline$\alpha_{i}$ & $\beta_{i}$ & $\gamma_{i}$ & $\eta_{i}$ & $k_{i}$ \\
\hline 0.025 & 1.35 & 22.983 & 0.5035 & 0.02075 \\
\hline 0.027 & 1.24 & 137.370 & 0.5773 & 0.02446 \\
\hline 0.015 & 0.80 & 363.704 & 0.4968 & 0.02270 \\
\hline 0.017 & 0.70 & 720.450 & 0.5634 & 0.03033 \\
\hline 0.007 & 0.60 & 1428.43 & 0.5244 & 0.03356 \\
\hline 0.009 & 0.50 & 2856.22 & 0.5978 & 0.0367 \\
\hline
\end{tabular}

TABLE III.

\begin{tabular}{|c|c|c|}
\hline $\begin{array}{c}\text { Fuel Cost in MR/hr } \\
\text { for Proposed Method }\end{array}$ & $\begin{array}{c}\text { Fuel Cost in MR/hr for Con- } \\
\text { ventional Method }\end{array}$ & Deviation \\
\hline 0.759 & 3.225 & -0.764 \\
\hline 0.656 & 0.6988 & -0.061 \\
\hline 0.126 & 0.1519 & -0.170 \\
\hline 0.143 & 0.1951 & -0.267 \\
\hline 0.121 & 0.206 & -0.412 \\
\hline 0.0348 & 0.150 & -0.0076 \\
\hline
\end{tabular}

Following the increased generation level of solar power plant the generation [1] cost of thermal power plant decreases. As a result of this deviation between cost of generation incorporating valve point loading for cost and emission function in the proposed method and the conventional method without valve point loading with respect to various power demands undergoes a change as reflected in Fig-3.

The IEEE 30 bus test case system used for the Present dissertation is shown in Fig-4.

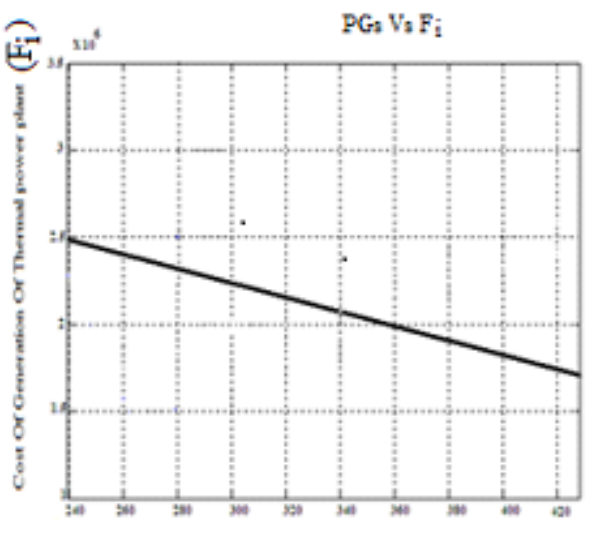

Real porer for the ith unit of the solar power plant(PGs)

Figure 1. Variation of real power of Solar power plant versus Cost of generation of thermal power plant

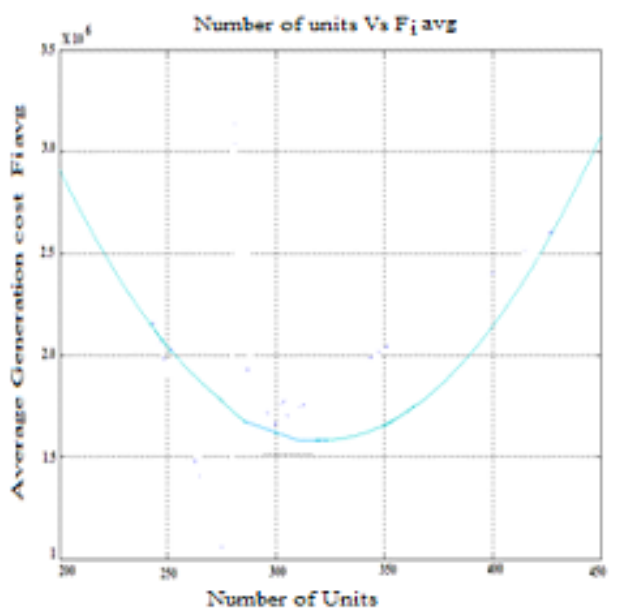

Figure 2. Number of units versus average generation cost

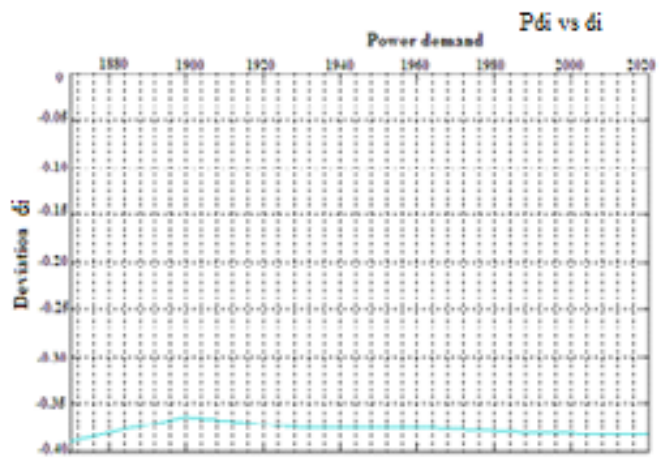

Figure 3. Power demand versus deviation 


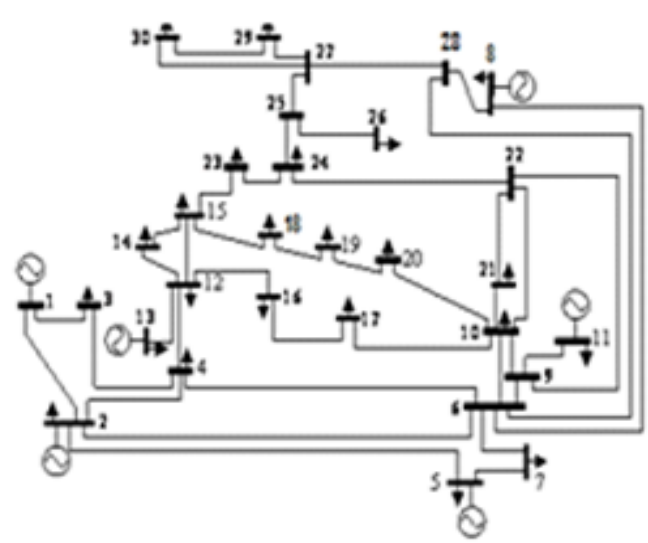

Figure 4. IEEE 30 bus test case system

\section{CONCLUSION}

Various soft computing methods like neural network, fuzzy logic etc. were used for optimal dispatch for optimizing the cost of generation of thermal power plant with multi-objectives[5] wherein the computation time for simulation and convergence of the result became cumbersome and sluggish. So at its favor the concept of hybrid solar thermal power plant (HSTP) owning quadratic programming [6] method, an emerging evolutionary programming technique that was involved in the current dissertation, found useful in regulating greenhouse gasses optimizing the emission level so as to maximize the real power generation by reducing the global warming level. The use of multi-objective generation dispatch incorporating the cost of generation and emission as well with nonsmooth cost and emission function with valve point loading and quadratic programming approach can be incorporated for reduction in emission level of SO2, CO, N2O and other greenhouse gasses so as to maximize real power generation level for yielding the optimum cost of generation.

\section{REFERENCES}

[1] AllenJ.Wood, Bruce F.WollenBerg, Power generation operation and control, Wiley India edition.

[2] C E Lin, G L ivianib; Hierarchical economic dispatch of piece wise quadratic cost functions.,IEEE transactions on PAS, Vol PAS -103 , No 6 June 1984

[3] Derong Liu, Ying Cai; Taguchi method for solving economic dispatch problem with non- smooth cost function. IEEE transactions on power systems,Vol.20, No. 4, Nov 2005. http://dx.doi.org/10.1109/TPWRS.2005.857939

[4] Kwang Y. Lee and Arthit Sode - Yome , June Ho Park; Adaptive Hop field nueral networks for economic load dispatch,IEEE transactions on power systems. Vol 13 No 2. May1998.
[5] N Rama raj, R. Raja Ram; Analytical approach to optimize generation schedule of plant with multiple fuel options. Journal of institution of engineers (India), Vol68. pt EL Dec 1987.

[6] Po-Hung Chen, Hong-Chan Chang; Large - scale economic dispatch by genetic algorithm transactions on power systems, Vol10, No 4, Nov1995.

[7] RMS Dana raj, Dr F.Ggajendran; An efficient algorithm to find optimal economic load dispatch for plants having discontinuous fuel cost functions., Journal of institution of engineers(India)., Vol 83,pt El dec 2004.

[8] S. Rajasekaran ,G.A Vijaya Lakshmi Pai, Nueral networks, Fuzzy Logic and Genetic Algorithms, Prentice Hall of India private Limited.

[9] Singiresu S .Rao Engineering Optimization theory and practice third edition,Newage international(p) Limited,Publishers.

\section{AUTHORS}

S. K. Dash (corresponsding author) received the Chartered Engineering from I, E, India in 1991 and M.E degree in electrical engineering from UCE, Burla (Sambalpur University), India, in 1998 and the Ph.D. degree from Utkal University, Odisha, India in the year 2006. He has been with the Electrical Engineering Department, Gandhi Institute for Technological Advancement as a Professor and Head of the Department since 2005. Prior to it he worked in industry for 5 years and in OSME, Keonjhar, for 2 years and in Krupajal Engineering College for 4 years. His research interests are power system planning, operation, and optimization techniques applied to power systems. Dr. Dash received Pandit Madan Mohan Malaviya award, Union Ministry Of Power Prize and gold medals there of for his research papers on Multi Objective Generation Dispatch. He too authored two books entitled 'Fundamentals Of Electromagnetic Field Theory' and 'Basic Electrical Engineering' under the umbrella of PHI Publication and YESDEE publication in the year 2010 and 2016 respectively. Dr. Dash is engaged as a reviewer of EPCS, and EPSR journals of IEEE. Contact: Dept. of Electrical Engineering, GITA, Bhubaneswar, Odessa India, Tel: +917894039770, hodeegita@gmail.com.

Priyambada Ray procured her B.Tech from SIET, Dhenkanal and continuing M.Tech at present in GITA Engineering College, Bhubaneswar, under the guidance of professor Dr. S.K. Dash. Contact: Dept. of Electrical Engineering, GITA, Bhubaneswar, Odessa India, Tel: +917790009284, priyambadaray5@gmail.com.

Chinmaya Kumar Panda procured his B.Tech from MIET, Bhubaneswar and continuing M.Tech at present in GITA Engineering College, Bhubaneswar, under the guidance of professor Dr. S.K. Dash. Contact: Dept. of Electrical Engineering, GITA, Bhubaneswar, Odessa India, Chinmaya.eee2010@gmail.com.

Submitted 30 March 2016. Published as resubmitted by the authors 15 June 2016. 